\title{
A Go Where You Look Tele-Autonomous Rough Terrain Mobile Robot
}

\author{
Ray Jarvis \\ Intelligent Robotics Research Centre \\ Monash University \\ P.O. Box 35, Victoria 3800 \\ AUSTRALIA \\ e-mail: Ray.Jarvis@eng.monash.edu.au
}

\begin{abstract}
This paper describes an extension of previous work on an eye gaze/ head tracking control of a four wheel drive, rough terrain wheelchair (for the 'adventurous' disabled), provided with three levels of control which permit a safe teleautonomous style navigation. Here a remote tele-autonomous mode is tested with an eye gaze/ head track system and experimental results reported. In essence, the four wheel drive wheelchair could be any rough terrain vehicle such as a bulldozer or excavation machine where the operator's hands can be freed for other teleoperated manipulation tasks. Alternatively, a disabled person with only eye gaze control could drive a robotic vehicle, say a lawn mower, from a remote location. Video sequences of the system in full operation will be shown at presentation.
\end{abstract}

\section{Introduction}

The notion of using head pose and eye gaze tracking for controlling a wheelchair is very attractive in that such a provision might enable a severely disabled user to move about with considerable independence despite the disablement. Providing some levels of protection from risk of collision and short term assistance regarding local safe passage simply adds to the attractiveness of this type of project.

Just such a system [1] has been developed using a rough terrain, fourwheel drive wheelchair for the 'adventurous' disabled to navigate on beaches, through forests and such. Clearly, in this application, which brings a number of robotic sensory and control tools to the service of a wheelchair user, the guiding intelligence is on-board, in the mind of the user.

It is not difficult to reconfigure the above scenario to that where the user is at a remote site and the wheelchair a teleoperated or tele-autonomous [2] (as will be explained later) robotic vehicle. The wheelchair, in this new situation, simply represents a rough terrain outdoor mobile robot. An excavator [3], heavy duty lawn-mower [4] or a number of other functional vehicles might equally substitute for the wheelchair, but the control structure would be the same.

Whilst it can be argued that head pose and eye gaze tacking can be used to provide a 'go where you look' mode of teleoperational steering and speed 
control which a severely disabled person may operate, it is more realistic to consider applications where leaving the hands (and feet) of an able bodied operator free to control other aspects of a task is of real advantage (as would be the case for the control of an excavator with an articulated bucket and a turret).

Of course, whilst a wheelchair user's head pose and eye gaze might direct the motion of the wheelchair in the context of what the user sees from onboard the vehicle, the remote user will need a video image from an on-board camera to do much the same.

This paper will report on the concepts behind, the implementation details of and the physical experiments undertaken with a 'go where you look' teleoperated rough terrain mobile robot (four-wheel drive wheelchair) using head pose and eye gaze tracking for high level guidance with two lower levels of control. These render the systems tele-autonomous, where some degree of local, short term path planning and collision avoidance relieves the top level guidance of some detailed control function and provides a safety net when poor judgment is acted upon. The system is supported by Global Positioning System localisation and a laser rangefinder for local environmental mapping.

The next section deals with the overall system. Then follows the description of and rationale behind the three level control strategy used. Next, the eye gaze/ head tracking system used for the 'go where you look' aspect of the project will be presented. The configuration of the remote control station will then be described. Trials to date will be presented. Finally, future work, discussion and conclusions close the paper.

\section{Overall System}

The overall system schematic is shown in Figure 1 and the instrumented robotic vehicle in Figure 2. The robotic vehicle used for this research is a $4 \times 4$ rough terrain wheelchair used in earlier projects. However, the following tele-autonomous strategy could easily have targeted other vehicles such as ride-on lawn mowers, bulldozers, excavation machines and so on. On-board the vehicle are a number of sensory, control, computational and communications resources. A Silicon Graphics workstation collects range data from an Erwin Sick laser rangefinder and sends control instructions via a dedicated microprocessor to the vehicle PID wheel velocity controller. The rangefinder is capable of collecting range data at $1 / 2$ degree intervals over a $180^{\circ}$ planer sweep up to 50 metres away at an accuracy of $\pm 3 \mathrm{~cm}$. In this experiment only 90 samples are taken at $2^{\circ}$ intervals. The vehicle is differentially steered with the right pair and left pair wheels controlled in unison. A passive Ackerman steering system on the front wheels makes for smooth non-skid forward turns but instability in reversing is the price paid for this advantage. 


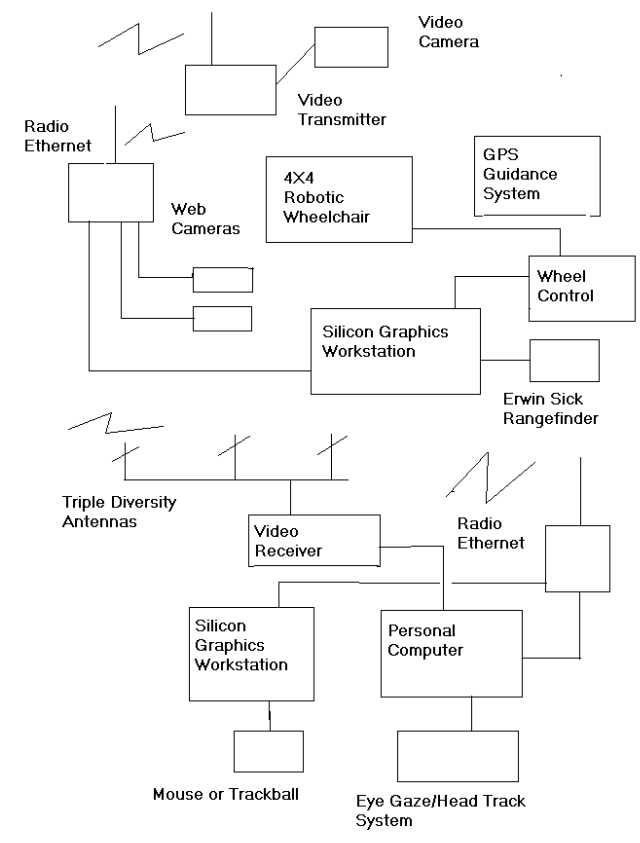

Fig. 1. Overall System Schematic

\section{Three Level Control System}

The idea of tele-autonomous control arose from the need for a less than perfect operator to be able to control the robotic vehicle within a safety net which minimises the risk of collision, yet at the same time gives the user maximum freedom within this protective envelope. This makes obvious sense when the operator is a disabled person on-board the vehicle. However, this also makes sense when the operator is able bodied but remote from the vehicle, as control can be achieved with less concentration on detail with the expectation that local operators will generally protect the vehicle in the event of operator misjudgement or distraction.

The top level control for this project is provided by the human operator, whose actions are considered to define 'user intention'. This 'intention' can be modified by the two lower levels of control. It is feasible to contemplate this top level control being provided by an artificially intelligent planner in due course. Figure 4 illustrates the general three level control philosophy. The deepest and lowest control level is pure reaction and can be configured to operate even if communication links with the remote control station are broken. For development purposes, however, it is convenient to remote log-in from the Silicon Graphics home-station to the computer on-board to monitor 


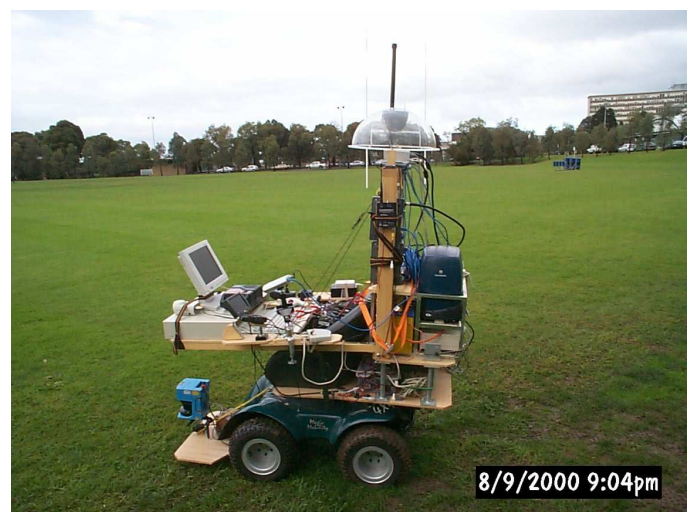

Fig. 2. Instrumented Mobile Robot (4x4 wheelchair)

Four cameras are on board. Two of these are web-cams with their own URLs. One of these is used to view a GPS navigation system screen [see Figure 3], the other can be adjusted manually to present any auxiliary view deemed useful. Another camera can be panned and tilted by a passenger (used for the genuinely wheel chair mode in previous research). The fourth camera is currently used to provide the remote operator with the forward looking view for eye gaze/ head track vehicle navigation.

details of the processing stages and control parameters actually issued. The reaction layer uses data from the Sick rangefinder to determine its actions. The vehicle is slowed to a stop (speed proportional to distance to obstacle) on approaching an obstacle in the front and veers away from side obstacles despite user intention to do otherwise. The reaction layer also informs the higher level systems of near misses to allow some measure of the competence of the operator to be gauged. This measure is used in calculating the mix of 'user intention' and 'local advice' used for the middle level control.

The middle level of control is the most innovative from a conceptual point of view. Range data from the laser rangefinder is analysed over an approximately $6 \mathrm{~m} \times 6 \mathrm{~m}$ area directly in front of the vehicle. Obstacles viewable from the location of the rangefinder are mapped onto a $32 \times 32$ cellular grid floor plan over this $6 \mathrm{~m} \times 6 \mathrm{~m}$ plot. A Distance Transform [5] is carried out over all free space, emanating out from all obstacle boundary cells so that each free cell is given an integer value equal to the number of steps it is away from its nearest obstacle. Clearly, centres of fairly open areas will have the larger numbers. Next, from the location of the rangefinder (considered to be at the bottom centre of the $32 \times 32$ forward grid covering the $6 \mathrm{~m} \times 6 \mathrm{~m}$ plot) radial scans at $2^{\circ}$ intervals from $30^{\circ}$ to $150^{\circ}$ and steps of 0.5 metres accumulate the values in the cells traversed until an obstacle in encountered, or a specified radial cell distance is exceeded. The largest accumulated number indicates the safest direction to go in a fairly simplistic way. Figure 5 shows a typical 


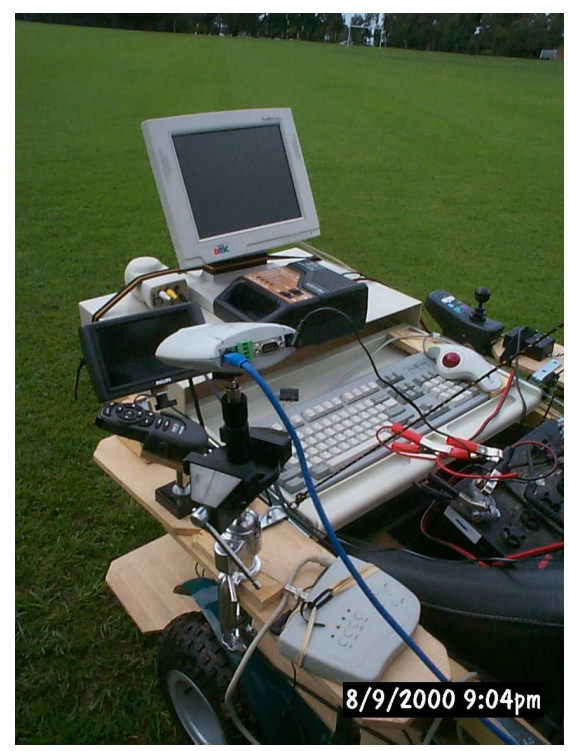

Fig. 3. Close-up showing GPS Screen and Web Cam

The web-cams and the on-board Silicon Graphics workstation are on a $10 \mathrm{mb}$ radio Ethernet $\mathrm{LAN}$; the vehicle can be up to $4 \mathrm{~km}$ away in lineof-sight from the remote station which is on a wired and radio Ethernet LAN. The main camera (used for gaze control) is linked to a high quality video transmitter which communicates to a multiple diversity antenna receiver at the remote workstation.

At the remote control workstation, a $\mathrm{PC}$ operating the gaze system and a Silicon Graphics workstation are on the LAN.

intensity coded Distance Transform and the optimal direction advised. The direction is termed the 'advised direction'. If the operator is considered totally incompetent, this sets the steering value. If the operator is considered totally competent, the 'advice' is ignored. In between these extremes a linear mix of user 'intention' and rangefinder devised 'advice' sets the steering. The analysis process repeats with cycles of less than 1 second. The mixing factor is determined by the user competency measure determined from using recent near miss and lack thereof counts to adjust this value down and up, respectively, between 0.0 and 1.0 .

In the case of a disabled person in the wheelchair, where competence may relate to fatigue or modes of disability, this personalised adaptive strategy makes reasonable sense. In the case of remote tele-autonomous operation by an able bodied user this strategy may need adjustment.

The three levels of control work together quite well, giving a nice mix of sensor mediated user 'intention', where the degree of autonomous control is inverse operator competence based, and also provides a fall back reaction 


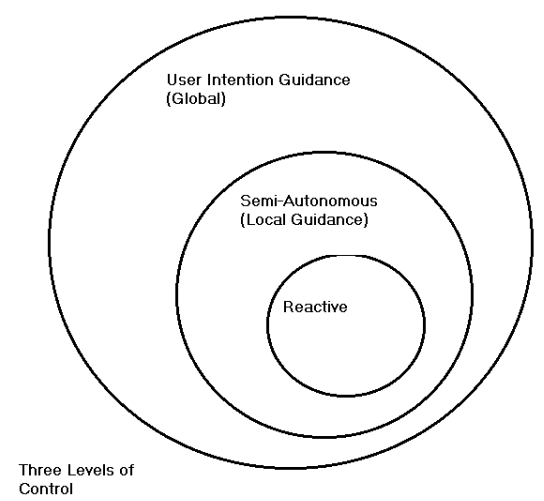

Fig. 4. Three Level Control Strategy

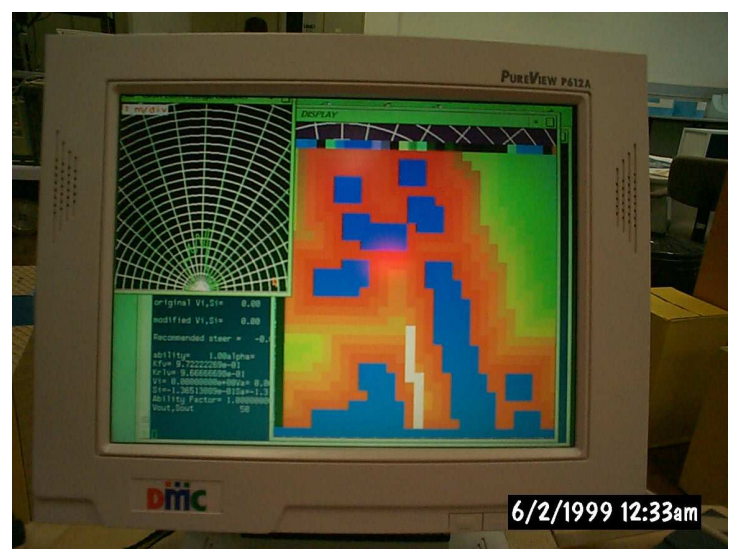

Fig. 5. Typical Local Distance Transform Based Steering 'Advice' 
based obstacle avoidance facility which may never be exercised but is there for safety.

The human level of control 'intention' can be provided by use of a mouse or trackball but in this paper, eye gaze/ head track data is substituted. However, reverting back to the mouse or trackball mode is simple and direct. As was mentioned earlier, the human top level control can be replaced by an AI based navigation planner which can concentrate on the navigation strategy, giving less attention to the details.

\section{Eye Gaze/ Head Tracking System}

Figure 6 shows the commercially available passive stereovision based eye gaze/ head tracking system. This system can be purchased from Seeing Machines [6]. A pair of monochrome cameras view the operator's head/ face from a slightly upward looking pose. An individual user calibrates the system by indicating the position of facial landmarks, including the corners of the eyes and mouth and perhaps also the nostrils, side burn edges and other distinguishing features. Using this data the system can calculate the $3 \mathrm{D}$ pose of the head, locate the eyes and determine the gaze direction relative to the head pose by tracking the locations of the pupils within the frame of the eyes. The gaze vector (pitch and yaw) can be calculated from these parameters. Figure 7 is a screen close-up showing a vector protruding from the bridge of the nose which indicates head orientation and vectors protruding out of the eyes which indicate gaze direction. The $\mathrm{x}, \mathrm{y}, \mathrm{z}$ lateral position of the head is also known. The eye gaze/ head pose data are generated at up to 60 times per second. This data can be logged on disk for future scrutiny or transmitted to a specified local area network location. The wearing of spectacles does degrade the quality of the measurements but not drastically so. Reasonably reliable operation is assured within a tolerance region for the location and pose of the head. Eye blink (closure) can also be measured. Blink data can be used for control purposes but this has not yet been done on this project. Some practise is needed for reliable smooth operation and eye fatigue can set in if the operator tries too hard to present a clear view of wide open eyes to the cameras. The system also indicates when eye tracking is being successfully achieved and when not.

\section{Remote Control Station}

Figure 8 shows the remote control station. The gaze system logs its parameters onto the workstation on-board the robot using the LAN and radio Ethernet link. For convenience, the on-board computer is remotely logged in from a Silicon Graphics workstation at the remote control station. This is not necessary but provides easy access to useful partial computation results and the steering 'advice' map. The gaze system is controlled by its own Pentium 


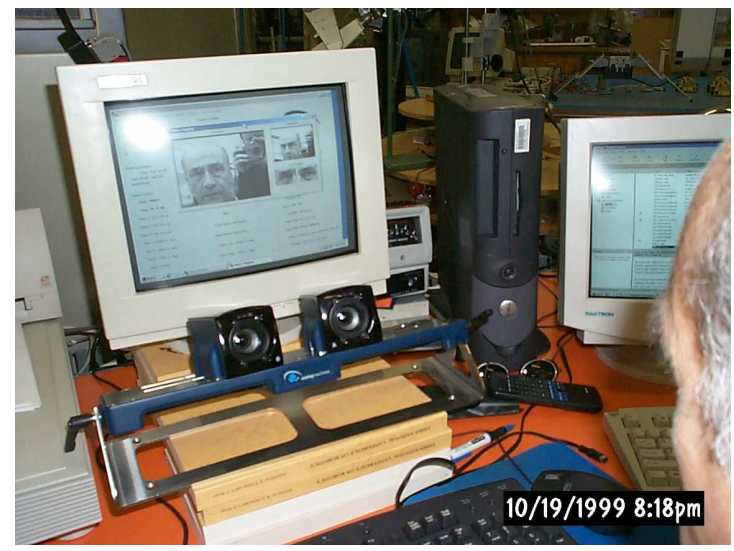

Fig. 6. Eye Gaze/ Head Tracking System

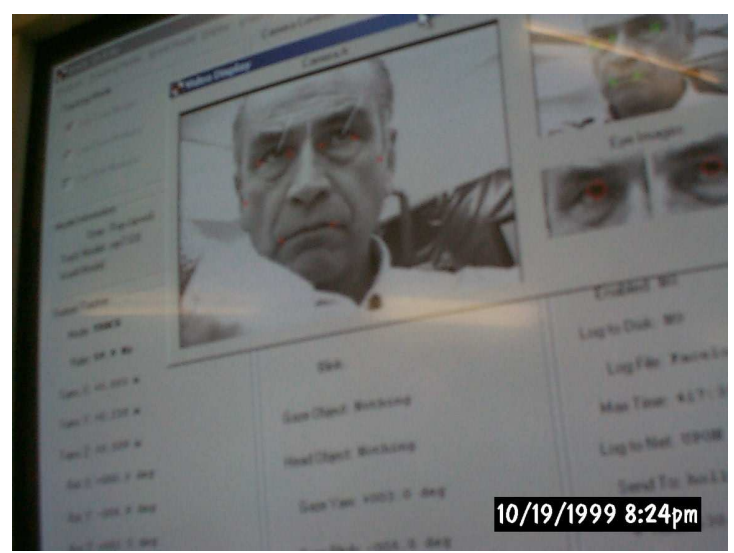

Fig. 7. Close-up of Eye Gaze/ Head Tracking Screen showing Head Pose and Eye Gaze Vectors

class PC. The monitor of this PC has been rigged so that one can switch it between being the gaze set-up screen (during initiation of the mission) and a video monitor viewing the scene from the robot mounted camera (during the mission). The video image quality is very high due to the use of a quality video transmitter and a multiple diversity antenna system on the receiver. The movement and orientation of the robot seems to have a minimal effect on the image quality.

Once the mission has begun the operator looks at the screen, gazing left and right for steering and down and up for speed. If the operator looks away the system nulls the gaze data and control reverts to the mouse or trackball 


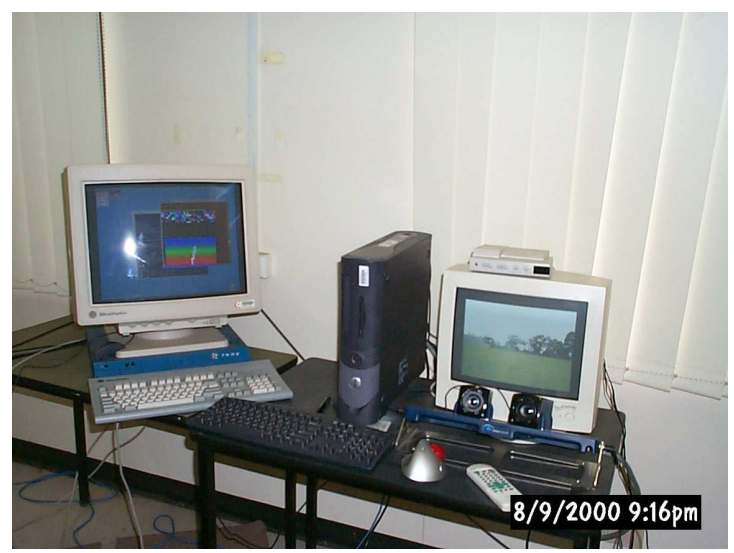

Fig. 8. Remote Teleoperation Base Station

which is typically centred or deliberately placed in a bias position in an additive way to the gaze data.

\section{Experimental Results}

The system has been trialed on a playing field with some contrived obstacles (coloured cardboard boxes). The three level control system works quite well but some care has to be taken in being consistent with regard to the position of the user in front of the gaze system. Some means of quick calibration for relating gaze values to intention still needs to be added. So far only relatively slow speed trials have been carried out.

The location of the robot can be observed by viewing the GPS map via a web-cam which updates its image at approximately 1 second intervals. Other localisation modes could also be added (e.g. rotating bar code scanning localiser for small excursions, long range radar or laser scanning for larger excursions).

The weakest part of the experiments so far is the need for some concentration whilst using the gaze system. The use of blink information is also not yet properly exploited.

\section{Future Work, Discussion and Conclusions}

The means by which one could use the gaze system with less commitment and in a more relaxed way needs some consideration. A quick recalibration setting of gain factors, exploitation of blink data for mode changes and some degree of smoothing may all contribute to this end. The extent to which the freed hands of the operator could be used for other control purposes may also 
be worth investigating. Perhaps the control of a boom arm and bucket on an excavating machine or a robotic manipulator used for picking up samples could be such cases.

However, the extent to which the use of gaze control is to be preferred to other modalities of control (e.g. voice, hand, feet, body gestures) is not obvious, except perhaps in some special circumstances.

One disadvantage of having to observe the eyes is that head mounted displays (HMD) can not be used with this system. The main advantage with respect to the HMD viewing alterative is that the gaze system is not only entirely passive but needs no specialised apparatus to be donned by the user.

Of interest is the ease with which the remote operation site can be anywhere on the network, so long as the gaze system is also at that site and the stationary radio Ethernet unit is within $4 \mathrm{~km}$ (line-of-sight) of the robotic vehicle.

In conclusion, this paper has presented a novel use of a passive eye gaze/ head track system for mobile robot navigation in the context of a three level control strategy where human intention can be modified to reduce the risk of collision. Whether this type of control has a strong future for this type of application is not yet understood.

\section{References}

1. Jarvis, R.A. A 'Go Where You are Looking' Semi-Autonomous Rough Terrain Robotic Wheelchair, accepted for presentation at the First International ICSC Congress on Autonomous Intelligent Systems, Deakin University, Geelong, Australia, 12-15 Feb. 2002.

2. Jarvis, R.A. Tele-Autonomous Watercraft Navigation, International Symposium on Experimental Robotics, Hawaii, Dec. 10 - $13^{\text {th }}$ 2000, pp. 539-546.

3. Jarvis, R.A. Sensor Rich Teleoperation of an Excavating Machine, Proc. Field and Service Robotics, Pittsburg, 29-31 Aug. 1999, pp. 238-243.

4. Jarvis, R.A. A Tele-Autonomous Heavy Duty Robotic Lawn Mower, Proc. (CD) Australian Conference on Robotics and Automation, 14,15 ${ }^{\text {th }}$ Nov. 2001, Sydney.

5. Jarvis, R.A. On Distance Transform Based Collision-Free Path Planning for Robot Navigation in Known, Unknown and Time-Varying Environments, invited chapter for a book entitled 'Advanced Mobile Robots' edited by Professor Yuan F. Zang World Scientific Publishing Co. Pty. Ltd. 1994, pp. 3-31.

6. Heinzmann, J., and Zelinsky, A. 3-D Facial Pose and Gaze Point Estimation using a Robust Real-Time Tracking Paradigm, Proc. International Conference on Automatic Face and Gesture Recognition, 1998, pp. 142-147. 